\title{
Quantitative ultrasound of os calcis BMD versus conventional DXA and peripheral QCT in interval assessment of BMD changes in adolescent females

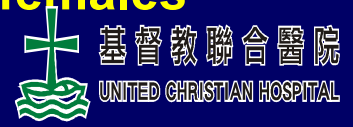

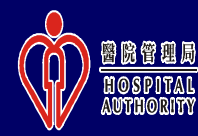

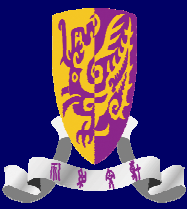

\author{
To WWK ${ }^{1}$ and $M$ WN Wong ${ }^{2}$
}

${ }^{1}$ Department of Obstetrics \& Gynaecology, United Christian Hospital

2 Department of Orthopaedics \& Traumatology, The Chinese University of Hong Kong, Hong Kong

Objective:To compare whether interval BMD changes in adolescent females that can be detected using conventional dual energy $X$-ray absorptiometry (DXA) of the lumbar spine and hip can also be detected using quantitative peripheral quantitative computerized tomography scans (pQCT) and quantitative ultrasound (QUS) of the os calcis

Methods:Two groups of adolescent females were recruited for assessment of BMD changes over an interval of 22- 24 months. These included full time collegiate dance students from a tertiary performing arts institute and healthy adolescents from an Adolescent Gynaecology clinic. Basic anthropometric measurements, baseline hormonal proffle, pelvic ultrasound, bio-impedance body fat estimation, DXA of lumbar spine and hip, PQCT of distal radius and tibia, QUS of os calcis were performed at first assessment, and DXA, pOCT and QUS were repeated at the second interval. Results: A total of 26 dance students and 14 nonexercising adolescents (mean age 18.6 years, range 16-19) were recruited. The dance students had lower body mass index (18.2 vs $19.2 \mathrm{~kg} / \mathrm{m} 2$, p $=0.03)$ and body fat percentage (19.1 vs $23.6 \%$, p $<0.005)$ compared to non-dancers. There were otherwise no significant differences in other basic anthropometric and baseline BMD measurements in the two groups. At the 24 month-assessment, DXA BMD values were consistently higher in both groups, though the increment was significantly greater in the dancers as compared to nondancers ( $\Delta$ lumbar spine $0.0758 \mathrm{Vs} 0.0329 \mathrm{~kg} / \mathrm{cm} 2$, $\mathrm{p}=0.006, \Delta$ neck of femur $0.046 \mathrm{Vs} 0.019 \mathrm{~kg} / \mathrm{cm} 2, \mathrm{p}=$ 0.004). QUS also showed a larger increment in dance students as compared to non-dancers $(\Delta$ soundness $18.1 \mathrm{Vs} 6.99, p=0.033 ; \Delta$ BMD $0.030 \mathrm{Vs}$ $0.01 \mathrm{~kg} / \mathrm{cm} 2, p=0.048)$. $\mathrm{PQCT}$ showed largely positive increments in both groups, but the magnitude was not significantly different between the two groups.

Conclusion: The findings confirmed that both adolescent dance students and non-dancers showed an increment in BMD values over the 24 month study interval. The differential increments were apparently better detected by conventional DXA as well as by QUS of the os calcis compared to pQCT measurements
Table 1. Comparing basic characteristics and initial assessment of dancers and non-exercising subjects

\begin{tabular}{|l|l|l|l|l} 
& $\begin{array}{l}\text { Dancers } \\
\mathrm{N}=26(\mathrm{SD})\end{array}$ & $\begin{array}{l}\text { Non-exercising } \\
\mathrm{N}=14(\mathrm{SD})\end{array}$ & $\begin{array}{l}\mathrm{p}- \\
\text { value }\end{array}$ & $\begin{array}{l}\text { Mean difference } \\
(95 \% \mathrm{CI})\end{array}$ \\
\hline Age & $18.3(1.41)$ & $18.6(0.74)$ & 0.47 & $\begin{array}{l}-0.30 \\
(-1.12 \text { to } 0.53)\end{array}$ \\
\hline Height $(\mathrm{cm})$ & $159.1(3.69)$ & $158.4(5.92)$ & 0.65 & $\begin{array}{l}0.69 \\
(-2.38 \text { to } 3.76)\end{array}$ \\
\hline Weight $(\mathrm{kg})$ & $46.1(3.52)$ & $48.5(6.58)$ & 0.14 & $\begin{array}{l}-2.39 \\
(-5.61 \text { to } 0.81)\end{array}$ \\
\hline BMI (kg/m²) & $18.2(1.18)$ & $19.2(1.90)$ & 0.03 & $\begin{array}{l}-1.05 \\
(-2.04 \text { to }-0.066)\end{array}$ \\
\hline Body Fat & $19.1(3.44)$ & $23.6(3.52)$ & $<0.001$ & $\begin{array}{l}-4.45 \\
(-0.677 \text { to }-2.12)\end{array}$ \\
\hline $\begin{array}{l}\text { Lumbar spine L2-L4 } \\
\left(\mathrm{g} / \mathrm{cm}^{2}\right)\end{array}$ & $0.921(0.089)$ & $0.968(0.134)$ & 0.19 & $\begin{array}{l}-0.046 \\
(-0.11 \text { to } 0.025)\end{array}$ \\
\hline $\begin{array}{l}\text { Mean neck of femur } \\
\left(\mathrm{g} / \mathrm{cm}^{2}\right)\end{array}$ & $0.903(0.077)$ & $0.874(0.136)$ & 0.38 & $\begin{array}{l}0.029 \\
(-0.038 \text { to } 0.097)\end{array}$ \\
\hline $\begin{array}{l}\text { Mean Ward's } \\
\left.\text { triangle }(\mathrm{g} / \mathrm{cm})^{2}\right)\end{array}$ & $0.746(0.092)$ & $0.768(0.142)$ & 0.57 & $\begin{array}{l}-0.021 \\
(-0.096 \text { to } 0.054)\end{array}$ \\
\hline $\begin{array}{l}\text { Mean Trochanter } \\
\left(\mathrm{g} / \mathrm{cm}^{2}\right)\end{array}$ & $0.753(0.081)$ & $0.719(0.122)$ & 0.30 & $\begin{array}{l}0.033 \\
(-0.031 \text { to } 0.099)\end{array}$ \\
\hline $\begin{array}{l}\text { Distal radius(core) } \\
\left(\mathrm{mg} / \mathrm{cm}^{3}\right)\end{array}$ & $274.04(68.4)$ & $272.68(78.17)$ & 0.95 & $\begin{array}{l}1.35 \\
(-45.96 \text { to } 48.67)\end{array}$ \\
\hline $\begin{array}{l}\text { Distal radius (total) } \\
\left(\mathrm{mg} / \mathrm{cm}^{3}\right)\end{array}$ & $610.16(59.46)$ & $598.68(53.62)$ & 0.53 & $\begin{array}{l}11.47 \\
(-25.91 \text { to } 48.87)\end{array}$ \\
\hline $\begin{array}{l}\text { Mean tibia (core) } \\
\left(\mathrm{mg} / \mathrm{cm}^{3}\right)\end{array}$ & $312.66(45.85)$ & $309.81(37.21)$ & 0.83 & $\begin{array}{l}2.85 \\
(-25 \text { to } 30.7)\end{array}$ \\
\hline $\begin{array}{l}\text { Mean tibia (total) } \\
\left(\mathrm{mg} / \mathrm{cm}^{3}\right)\end{array}$ & $625.54(133)$ & $602.43(94)$ & 0.55 & $\begin{array}{l}23.1 \\
(-55 \text { to } 101)\end{array}$ \\
\hline
\end{tabular}

Table 2. Comparison of interval change after $\mathbf{2 4}$ months between exercising and non-exercising subjects

\begin{tabular}{|c|c|c|c|c|}
\hline & $\begin{array}{l}\text { Dancers } \\
\mathrm{N}=26 \text { (SD) }\end{array}$ & $\begin{array}{l}\text { Non-xercising } \\
\mathrm{N}=14 \text { (SD) }\end{array}$ & $\mathrm{p}$-value & $\begin{array}{l}\text { Mean difference } \\
(95 \% \mathrm{Cl})\end{array}$ \\
\hline $\begin{array}{l}\text { Weight change } \\
(\mathrm{kg})\end{array}$ & $-0.38(1.06)$ & 0 (1.03) & 0.27 & $\begin{array}{l}-0.38 \\
-(-1.09 \text { to } 0.31)\end{array}$ \\
\hline $\begin{array}{l}\text { BMl change } \\
(\mathrm{kg} / \mathrm{m} 2)\end{array}$ & $-0.155(0.423)$ & $0.031(0.441)$ & 0.18 & $\begin{array}{l}-0.187 \\
(-0.469 \text { to } 0.094)\end{array}$ \\
\hline Body fat change $\%$ & $-0.107(2.24)$ & $-1.92(2.55)$ & 0.025 & $\begin{array}{l}1.82 \\
(0.241 \text { to } 3.4)\end{array}$ \\
\hline $\begin{array}{l}\text { Lumbar L2-L4 } \\
\left(\mathrm{g} / \mathrm{cm}^{2}\right)\end{array}$ & \begin{tabular}{|l|}
0.0758 \\
$(0.0523)$ \\
\end{tabular} & $0.0179(0.0239)$ & $<0.001$ & $\begin{array}{l}0.057 \\
(0.027 \text { to } 0.088)\end{array}$ \\
\hline $\begin{array}{l}\text { Neck of femur } \\
\left(\mathrm{g} / \mathrm{cm}^{2}\right)\end{array}$ & $0.0459(0.0307)$ & $0.0098(0.0111)$ & $<0.001$ & $\begin{array}{l}0.036 \\
(0.018 \text { to } 0.053)\end{array}$ \\
\hline $\begin{array}{l}\text { Ward's triangle } \\
\left(\mathrm{g} / \mathrm{cm}^{2}\right)\end{array}$ & $\begin{array}{l}0.0369 \\
(0.0247)\end{array}$ & $0.0094(0.015)$ & 0.001 & $\begin{array}{l}0.0275 \\
(0.012 \text { to } 0.042)\end{array}$ \\
\hline $\begin{array}{l}\text { QUS os calcis } \\
\text { Soundness }\end{array}$ & 18.1 10.6) & $6.99(21.4)$ & 0.033 & $\begin{array}{l}11.1 \\
\text { (0.93 to } 21.37)\end{array}$ \\
\hline $\begin{array}{l}\text { QUS ox calcis } \\
\text { BMD }\left(\mathrm{g} / \mathrm{cm}^{2}\right)\end{array}$ & $0.0306(0.031)$ & $0.01(0.027)$ & 0.048 & $\begin{array}{l}0.020 \\
(0.0001 \text { to } 0.04)\end{array}$ \\
\hline Trochanter $\left(\mathrm{g} / \mathrm{cm}^{2}\right)$ & \begin{tabular}{|l|}
0.0262 \\
$(0.0314)$
\end{tabular} & $0.0129(0.0114)$ & 0.13 & \begin{tabular}{|l|}
0.013 \\
$(-0.004$ to 0.031$)$
\end{tabular} \\
\hline $\begin{array}{l}\text { Distal radius(core) } \\
\left(\mathrm{mg} / \mathrm{cm}^{3}\right)\end{array}$ & $12.76(10.57)$ & $12.57(8.24)$ & 0.95 & $\begin{array}{l}0.197 \\
(-6.4 \text { to } 6.8)\end{array}$ \\
\hline $\begin{array}{l}\text { Distal radius (total) } \\
\left(\mathrm{mg} / \mathrm{cm}^{3}\right)\end{array}$ & $19.30(17.35)$ & $23.28(20.30)$ & 0.51 & $\begin{array}{l}-3.97 \\
(-16.33 \text { to } 8.38)\end{array}$ \\
\hline $\begin{array}{l}\text { Mean tibia (core) } \\
\left(\mathrm{mg} / \mathrm{cm}^{3}\right)\end{array}$ & $11.3(8.24)$ & $13(5.9)$ & 0.49 & $\begin{array}{l}-1.72 \\
(-6.77 \text { to } 3.32)\end{array}$ \\
\hline $\begin{array}{l}\text { Mean tibia (total) } \\
\left(\mathrm{mg} / \mathrm{cm}^{3}\right)\end{array}$ & 19.19 (13.44) & 22.78 (13.99) & 0.43 & $\begin{array}{l}-3.59 \\
(-12.74 \text { to } 5.55)\end{array}$ \\
\hline
\end{tabular}

